\title{
THE
}

\section{ROBUST POPULATION MANAGEMENT UNDER UNCERTAINTY FOR STRUCTURED POPULATION MODELS}
A. Deines
E. Peterson
D. Boeckner
J. Boyle
A. Keighley

See next page for additional authors

Follow this and additional works at: https://digitalcommons.uri.edu/math_facpubs

Terms of Use

All rights reserved under copyright.

\section{Citation/Publisher Attribution}

Deines, A. , Peterson, E. , Boeckner, D. , Boyle, J. , Keighley, A. , Kogut, J. , Lubben, J. , Rebarber, R. , Ryan, R. , Tenhumberg, B. , Townley, S. and Tyre, A. J. (2007), ROBUST POPULATION MANAGEMENT UNDER UNCERTAINTY FOR STRUCTURED POPULATION MODELS. Ecological Applications, 17: 2175-2183. doi:10.1890/06-1090.1

Available at: https://doi.org/10.1890/06-1090.1

This Article is brought to you for free and open access by the Mathematics at DigitalCommons@URI. It has been accepted for inclusion in Mathematics Faculty Publications by an authorized administrator of DigitalCommons@URI.For more information, please contact digitalcommons-group@uri.edu. 


\section{Authors}

A. Deines, E. Peterson, D. Boeckner, J. Boyle, A. Keighley, J. Kogut, J. Lubben, R. Rebarber, R. Ryan, B. Tenhumberg, S. Townley, and A. J. Tyre 


\title{
ROBUST POPULATION MANAGEMENT UNDER UNCERTAINTY FOR STRUCTURED POPULATION MODELS
}

\author{
A. Deines, ${ }^{1}$ E. Peterson, ${ }^{2}$ D. Boeckner,${ }^{3}$ J. Boyle,${ }^{4}$ A. Keighley, ${ }^{5}$ J. Kogut,${ }^{6}$ J. Lubben, ${ }^{3}$ R. Rebarber,${ }^{3}$ \\ R. Ryan, ${ }^{7}$ B. Tenhumberg,${ }^{8}$ S. Townley, ${ }^{9}$ and A. J. Tyre ${ }^{8,10}$ \\ ${ }^{1}$ Department of Mathematics, Kansas State University, Manhattan, Kansas 66506 USA \\ ${ }^{2}$ Department of Mathematics, Wittenberg University, Springfield, Ohio 45501 USA \\ ${ }^{3}$ Department of Mathematics, University of Nebraska-Lincoln, Lincoln, Nebraska 68588 USA \\ ${ }^{4}$ Department of Mathematics, University of Notre Dame, Notre Dame, Indiana 46556 USA \\ ${ }^{5}$ Department of Mathematical Sciences, Rensselaer Polytechnic Institute, Troy, New York 12180 USA \\ ${ }^{6}$ Department of Mathematics, Simmons College, Boston, Massachusetts 02115 USA \\ ${ }^{7}$ Department of Mathematics, University of Rhode Island, Kingston, Rhode Island 02881 USA \\ ${ }^{8}$ School of Natural Resources, University of Nebraska-Lincoln, Lincoln, Nebraska 68588 USA \\ ${ }^{9}$ Department of Mathematical Sciences, University of Exeter, Exeter EX4 4QE United Kingdom
}

\begin{abstract}
Structured population models are increasingly used in decision making, but typically have many entries that are unknown or highly uncertain. We present an approach for the systematic analysis of the effect of uncertainties on long-term population growth or decay. Many decisions for threatened and endangered species are made with poor or no information. We can still make decisions under these circumstances in a manner that is highly defensible, even without making assumptions about the distribution of uncertainty, or limiting ourselves to discussions of single, infinitesimally small changes in the parameters. Suppose that the model (determined by the data) for the population in question predicts long-term growth. Our goal is to determine how uncertain the data can be before the model loses this property. Some uncertainties will maintain long-term growth, and some will lead to long-term decay. The uncertainties are typically structured, and can be described by several parameters. We show how to determine which parameters maintain long-term growth. We illustrate the advantages of the method by applying it to a Peregrine Falcon population. The U.S. Fish and Wildlife Service recently decided to allow minimal harvesting of Peregrine Falcons after their recent removal from the Endangered Species List. Based on published demographic rates, we find that an asymptotic growth rate $\lambda>1$ is guaranteed with $5 \%$ harvest rate up to $3 \%$ error in adult survival if no two-year-olds breed, and up to $11 \%$ error if all two-year-olds breed. If a population growth rate of $3 \%$ or greater is desired, the acceptable error in adult survival decreases to between $1 \%$ and $6 \%$ depending of the proportion of two-year-olds that breed. These results clearly show the interactions between uncertainties in different parameters, and suggest that a harvest decision at this stage may be premature without solid data on adult survival and the frequency of breeding by young adults.
\end{abstract}

Key words: elasticity; Falco peregrinus anatum; matrix sensitivity; Peregrine Falcons; robustness; structured population models.

\section{INTRODUCTION}

Decision making under uncertainty is a pervasive characteristic of conservation biology. Sometimes, the scientific uncertainty can be so severe that it paralyzes decision making, or causes decisions to be made solely on social grounds, without being informed by science. Current quantitative approaches to decision making usually rely on being able to construct models or scenarios that illuminate the consequences of decisions for various stakeholders. Managers of wildlife populations use population projection matrices (Caswell 2001)

Manuscript received 26 June 2006; revised 26 February 2007; accepted 29 March 2007; final version received 10 May 2007. Corresponding Editor: T. R. Simons.

${ }^{10}$ Corresponding author.

E-mail: atyre2@unlnotes.unl.edu to assess decisions with increasing frequency, but parameters in these matrices are inherently uncertain. Unfortunately, the standard tools for assessing the effects of parameter uncertainty on matrix models require better data than is typically available in the management of threatened or endangered species. The method of sensitivity and elasticity analyses is predicated on analyzing perturbed behaviors resulting from small deviations away from some assumed nominal behavior. In fact, this approach can be misleading for large perturbations (see Mills et al. 1999, Hodgson and Townley 2004). Another standard approach is to use Monte Carlo simulations, where the data is assumed to be substantial enough to determine parameter estimates of the distributional form of random variables. In the management of threatened or endangered species, where information can be extremely scarce, it is unlikely that 
the perturbations are small, and in many cases there is not enough information available to know the distribution of uncertainties. Scarcity of data particularly impacts estimates of the variance, possibly leading to underestimates of the probability of extreme values. Even when empirical variance estimates or bounds on parameters are available, and accepted by all parties, correlations between parameters are certainly present and usually unknown. In this paper, we present an alternate approach for the systematic analysis of the effect of uncertainties on long-term population growth or decay. This approach does not require the perturbations to be small, can handle simultaneous uncertainty in several parameters, and does not require strong distributional assumptions.

Suppose that the model (determined by the data) for the population in question predicts long-term growth. Our focus is to determine how uncertain the data can be before the model loses this property. Roughly speaking, the robustness of a desired property (such as long-term population growth) to uncertainty or perturbation of data is a measure of how much the data can be changed before the desired property is destroyed. A general framework of robustness analysis, which has been developed in the field of control theory, has been adapted for population dynamics in ecology by Hodgson and Townley (2004). The robustness approach adopts a different viewpoint to that typified by sensitivity/elasticity analysis: the latter is microscopic, perturbing away from a nominal model and focusing in on the infinitesimal dependence of a specific dynamical property on the perturbation; the latter is essentially macroscopic and focuses in on perturbation as a function of required dynamical property. Hodgson and Townley (2004) tabulates a clear comparison between these micro- vs. macroscopic approaches.

While we build on the approach in Hodgson and Townley (2004), our approach differs from theirs in several ways. Most importantly our focus is on robustness of population growth (at least one eigenvalue greater than one in modulus), which is more delicate than their simpler problem of robustness of population decline (all eigenvalues less than one in modulus). In addition, we describe all acceptable uncertainties, while they give the answer in terms of the stability radius, which gives a distance that the data can be changed before causing the desired property to be lost.

The methods presented here are generalizable to all population projection matrices, but we illuminate the method with a particular problem: the decision to allow limited harvesting of a recently recovered endangered species. Peregrine Falcons (Falco peregrinus anatum) were placed on the endangered species list in 1970 (U.S. Fish and Wildlife Service 2003), due to DDT, habitat loss, hunting, and other factors. In addition to the ban on DDT, the implementation of fostering, hacking (young falcons slowly reintroduced to the wild in stages), and the release of over 6000 Peregrines helped populations recover (Craig et al. 2004).

With over 2000 breeding pairs in the United States, the population is again increasing, and falcons were removed from the endangered species list in 1999. There is renewed interest in harvesting Peregrine Falcons for falconry, and, in May 2001, the U.S. Fish and Wildlife Service allowed states west of the $100^{\circ}$ longitude line (from North Dakota through Texas) to allow harvesting of up to $5 \%$ of their state's population (U.S. Fish and Wildlife Service 2001). Falconers as a group have considerable interest in the outcome, as they contributed a huge, voluntary effort to foster and hack young birds during the recovery phase. For them, the new harvest permits are the payoff of a long and significant investment.

In July 2005, controversy arose over the number of falcons currently being harvested in Oregon. The Audubon Societies of Portland and Denver, the Center for Biological Diversity, and the New Mexico Audubon Council questioned the decision of the U.S. Fish and Wildlife Service allowing harvesting of the Peregrine Falcon population. In particular, the plaintiffs claimed that the U.S. Fish and Wildlife Service's calculations of the margin of error misrepresented the data, and consequently harvesting exposed Peregrine Falcon populations to unnecessary risk of decline. These concerns were dismissed and $5 \%$ of the population are still allowed to be harvested. ${ }^{11}$ The key issue on which this case hinged was whether or not the incorporation of uncertainty into the calculations of the allowable harvest rate was done appropriately.

After we apply our methods to the model for Peregrine Falcon population growth, we incorporate harvest effects into the population model to assess how different levels of harvesting reduce the robustness to uncertainty. How much uncertainty is tolerable is a value judgment, but the methods used in this paper make direct connections between uncertainty and maintenance of population growth under different management choices, without assuming that uncertainties are tiny or that errors have particular distributions.

\section{Methods}

\section{General method for classifying perturbations}

Begin by assuming that $\mathbf{A}$ is a time-invariant population projection matrix for the population in question. The leading eigenvalue of $\mathbf{A}$, which we denote by $\lambda(\mathbf{A})$, satisfies $\lambda(\mathbf{A})>1$, which implies that the population is increasing if $\mathbf{A}$ accurately models the population dynamics. The parameters used in this matrix are estimated from the available data, and are referred to as the nominal values, and $\mathbf{A}$ is referred to as the nominal matrix. The actual values of the parameters

11 Audubon Society of Portland v. United States Fish and Wildlife Service, No. 04-670-KI (D. Oregon July 21, 2005). 
could differ by unknown amounts from the nominal values, due to data collection errors and changes over time, so the actual population may not in fact be growing. We will explore the effects of this uncertainty on the population. It is not difficult to determine how far a single parameter can be perturbed before the population experiences negative population growth; one method is given in Appendix A. However, it is more difficult to determine the effect of independent perturbations of two or more underlying parameters. It is our goal to determine which combinations of perturbations maintain population increase, and which lead to population decline.

We denote the actual population projection matrix by $\tilde{\mathbf{A}}$, and we write

$$
\tilde{\mathbf{A}}=\mathbf{A}+\mathbf{P}
$$

where $\mathbf{P}$ is called the perturbation matrix. We do not know $\mathbf{P}$, and hence do not know $\tilde{\mathbf{A}}$ exactly. The nonzero entries of $\mathbf{P}$ correspond to the uncertain entries of $\mathbf{A}$. If the actual matrix is close to the nominal matrix (i.e., the data is accurate), then the entries of $\mathbf{P}$ will be small, but this is not guaranteed. The long-term population growth rate is directly determined by $\lambda(\tilde{\mathbf{A}})$, which we denote by $\lambda$.

If the dimension of the population vector is $n$, then the matrices $\mathbf{A}, \tilde{\mathbf{A}}$, and $\mathbf{P}$ have $n^{2}$ entries. The uncertainties are typically structured, and can be described by $m$ parameters $\left(p_{1}, p_{2}, \ldots, p_{m}\right)$, where $m \leq n^{2}$. The smaller the number of parameters we consider, the more tractable the analysis will be, so this approach will be easier if we consider only the most significant parameters, for instance, the parameters that affect $\lambda$ the most, or the most uncertain parameters. We say that $\left(p_{1}, p_{2}\right.$, $\left.\ldots, p_{m}\right)$ is admissible if $\mathbf{A}+\mathbf{P}$ is an acceptable projection matrix, and we let $S$ be the set of admissible $\left(p_{1}, p_{2}, \ldots\right.$, $\left.p_{m}\right)$; for example, it will be typical to restrict the perturbations so that the sum of the survival probabilities are always between 0 and 1 . We can denote the explicit dependence of $\tilde{\mathbf{A}}$ and $\lambda$ on $\left(p_{1}, p_{2}, \ldots, p_{m}\right)$ by writing

$$
\tilde{\mathbf{A}}=\tilde{\mathbf{A}}\left(p_{1}, p_{2}, \ldots, p_{m}\right), \quad \lambda=\lambda\left(p_{1}, p_{2}, \ldots, p_{m}\right) .
$$

Now consider the subset of $S$ given by

$$
C=\left\{\left(p_{1}, p_{2}, \ldots, p_{m}\right) \in S \mid \lambda\left(p_{1}, p_{2}, \ldots, p_{m}\right)=1\right\} .
$$

This is the set of $\left(p_{1}, p_{2}, \ldots, p_{m}\right)$ that lead to a leading eigenvalue of 1 . Mathematically, this set is a hypersurface. If we are considering two uncertain parameters, then $m=2$ and $C$ is a curve; this is the case which is illustrated in this paper. If we are considering three uncertain parameters, then $m=3$ and $C$ is an ordinary surface (that is, a two dimensional object in three dimensions). When $m=2$ or 3 , it is clear what it means for a particular $\left(p_{1}, p_{2}, \ldots, p_{m}\right)$ to be on one side or another of $C$. For hypersurfaces in dimensions higher than 3 , it is sometimes not possible to define the notion of the "side" of the hypersurface. However, for the surfaces described by Eq. 1, the notion of the side of $C$ can be made precise mathematically, using Proposition A.1 in Appendix A. Since we are assuming that the unperturbed matrix $\mathbf{A}$ has $\lambda(\mathbf{A})=\lambda(0,0, \ldots, 0)>1$, the "population growth" side of $C$ is the one containing ( 0 , $0, \ldots, 0)$. Hence, we consider all "good" perturbations to be those that are on the population growth side of $C$. Since the nominal model corresponds to $\left(p_{1}, p_{2}, \ldots, p_{m}\right)$ $=(0,0, \ldots, 0)$, one measure of robustness is how far $(0$, $0, \ldots, 0)$ is from $C$. In the case where $m=2$ or 3 , we get stronger results, since we get a graphical representation showing exactly which combinations of uncertainties maintain and destroy population growth.

If we are concerned with maintaining a particular growth rate, say $3 \%$, then we would replace $C$ with

$$
C_{1.03}=\left\{\left(p_{1}, p_{2}, \ldots, p_{m}\right) \in S \mid \lambda\left(p_{1}, p_{2}, \ldots, p_{m}\right)=1.03\right\} .
$$

Furthermore, it should be pointed out that for some applications we will be interested in maintaining population decay, in which case the good perturbations will be on the side of $C$ that guarantees that $\lambda\left(p_{1}, p_{2}, \ldots\right.$, $\left.p_{m}\right)<1$.

It still remains to find an equation for $C$. It is easy to find the hypersurface on which some eigenvalue of $\tilde{\mathbf{A}}$ is 1. Letting $I$ denote the $n \times n$ identity matrix, this hypersurface is

$$
\Gamma=\left\{\left(p_{1}, p_{2}, \ldots, p_{m}\right) \in S \mid \operatorname{det}\left[I-\mathbf{A}\left(p_{1}, p_{2}, \ldots, p_{m}\right)\right]=0\right\} .
$$

For the Peregrine Falcon model, in Appendix B we determine $\Gamma$ manually, and we show that $\Gamma$ is the same curve as $C$ by using an analytical argument based on the Peron-Frobenius Theorem (Seneta 1981). The manual computations would be arduous for larger matrices or multidimensional perturbations, so in the Supplement we provide MATLAB code demonstrating how to apply this method to a larger matrix and more complex perturbations. For all matrices we have tried so far, it is easy to confirm numerically that $\Gamma$ is the same curve as $C$. A thorough theoretical study of when $C=\Gamma$ is forthcoming (D. Boeckner, J. Lubben, R. Rebarber, B. Tenhumberg, and S. Townley, unpublished manuscript). Even if $\Gamma$ is not the same as $C$ (or cannot be proved to be the same as $C)$, it is still useful. For $\left(p_{1}, p_{2}, \ldots, p_{m}\right)$ on $\Gamma$, the eigenvalue of largest modulus $\lambda\left(p_{1}, p_{2}, \ldots, p_{m}\right)$ must be greater than or equal to 1 , since some eigenvalue of $\tilde{\mathbf{A}}\left(p_{1}, p_{2}, \ldots, p_{m}\right)$ is equal to 1 . Hence for $\left(p_{1}, p_{2}, \ldots, p_{m}\right)$ on side of $\Gamma$ which contains $(0,0, \ldots, 0)$, it is guaranteed that $\lambda\left(p_{1}, p_{2}, \ldots, p_{m}\right)>1$; however, it is not guaranteed that on the other side of $\Gamma$ we have $\lambda\left(p_{1}, p_{2}, \ldots, p_{m}\right)<1$.

\section{Falcon population model}

In this section, we consider a model for an endangered Peregrine Falcon population, and show how different kinds of uncertainties can be simultaneously, and globally, analyzed. We use a standard age-structured 
TABle 1. Nominal, or unperturbed, matrix parameters for the falcon model, sensitivities, and elasticities.

\begin{tabular}{llccc}
\hline \hline Parameter & \multicolumn{1}{c}{ Meaning } & Estimate & Sensitivity & Elasticity \\
\hline$F$ & no. nestlings fledged per pair & 1.660 & 0.0954 & 0.1539 \\
$R$ & proportion of female nestlings & 0.500 & 0.3166 & 0.1539 \\
$S_{0}$ & survival of nestling to age 1 & 0.544 & 0.2910 & 0.1539 \\
$S_{1}$ & survival of 1-yr-old birds & 0.670 & 0.2363 & 0.1539 \\
$S_{2}$ & survival of birds $\geq$ 2 yr old & 0.800 & 0.8901 & 0.6922 \\
$B$ & proportion of 2-yr-old birds that breed & 0 & 0.0453 & 0 \\
\hline
\end{tabular}

Note: These are "lower level" sensitivities, so the corresponding elasticities do not add to 1.

population projection model (Caswell 2001) with three age classes: birds less than one year old, birds older than one year and less than or equal to two years old, and birds older than two years. We refer to the population of birds in each of these three classes as $x_{1}, x_{2}$, and $x_{3}$, respectively, and the population vector is as follows:

$$
\mathbf{x}=\left(\begin{array}{l}
x_{1} \\
x_{2} \\
x_{3}
\end{array}\right)
$$

The population vector during year $k$ is denoted $\mathbf{x}_{k}$, and $\left(\mathbf{x}_{k}\right)_{k=0}^{\infty}$ satisfies the discrete time equation

$$
\mathbf{x}_{k+1}=\mathbf{A} \mathbf{x}_{k}
$$

where $\mathbf{A}$ is the population projection matrix. The nominal population projection matrix we use is a correction of the post-breeding model derived in Craig et al. (2004); the published matrix incorrectly includes an additional juvenile age class, although the reported model results are from the correct model (G. C. White, personal correspondence). The model parameters are $S_{0}$, the survivorship from birth to age one; $S_{1}$, the survivorship from age one to age two; $S_{2}$, the yearly survivorship for all older birds. The fecundity $F$ is assumed to be the same for all breeding pairs. Birds under two years old may or may not breed. We quantify this by letting $B$ represent the proportion of birds in the second age class that breed. $R$ denotes the proportion of birds that are female. In terms of these parameters, the nominal population projection matrix is

$$
\mathbf{A}=\left(\begin{array}{ccc}
0 & F R B S_{1} & F R S_{2} \\
S_{0} & 0 & 0 \\
0 & S_{1} & S_{2}
\end{array}\right)
$$

We use parameter values estimated from the Peregrine Falcons in Colorado, USA (Table 1; Craig et. al. 2004). We need to incorporate harvesting into the population projection matrix. We introduce the variable $h$, which represents the proportion of nestlings harvested, so the term $(1-h)$, denoting the proportion of nestlings remaining in the wild population, is included in the matrix A by multiplying this term by the fecundities (Caswell 2001). The amount of harvesting is assumed to be the same in both age classes since for many birds the age cannot be determined. This also assumes that the two age classes are equally vulnerable to harvesting. Let

$$
A_{h}=\left[\begin{array}{ccc}
0 & (1-h) F R B S_{1} & (1-h) F R S_{2} \\
S_{0} & 0 & 0 \\
0 & S_{1} & S_{2}
\end{array}\right] .
$$

Harvesting can affect the nesting habits of the parents and the survivorship of the remaining nestlings. Peregrine Falcons are known to re-nest (lay another clutch) if a clutch is lost early (Ratcliffe 1993). However, by U.S. Fish and Wildlife Service regulations, nestlings may not be harvested prior to 10 days of age (U.S. Fish and Wildlife Service 2001); thus removing nestlings will not cause the parents to re-nest. Removing a nestling could increase the survivorship of remaining nestlings due to less work for the parents. However, removing nestlings only minimally improves the survivorship of the remaining young (T. Cade, personal communication), thus, in modeling the worst case we may ignore this.

The U.S. Fish and Wildlife Service found $\lambda=1.03$ (Audubon Society of Portland v. United States Fish and Wildlife Service 2005), indicating long-term growth of $3 \%$. This is consistent with our nominal model, which has largest eigenvalue 1.0288. However, much of the data in $\mathbf{A}$ is uncertain.

\section{Data uncertainties}

For the purpose of demonstrating the method, we will focus on the two parameters contributing the most to the uncertainty of $\lambda$. We choose one of the parameters to be the most uncertain one, and the other parameter to be the one that affects the long-term population growth rate $\lambda$ the most.

We note that $B$ is completely unknown, and varies substantially between different populations. If a population is close to carrying capacity, then two-year-old birds are less likely to find a nesting site and so are less likely to breed (Hunt 1988). However, if the population is growing, then a high percentage of two-year-old birds will breed as there is less competition. Hence, we consider $B$ to be the most uncertain of the parameters.

In Fig. 1, we see how $\lambda$ is affected by changes in each of the parameters. When determining the effect of a parameter on $\lambda$, we can think of $\lambda$ as a function of each parameter while the other parameters stay fixed at the nominal values. Fig. 1 gives $\lambda(p)$ for each parameter. The value of $p$ (shown on the $x$-axis) represents the proportional change in the parameter from the nominal value (e.g., $p=-0.1$ represents a $10 \%$ decrease in the parameter). The $y$-axis gives the value of $\lambda$ obtained 


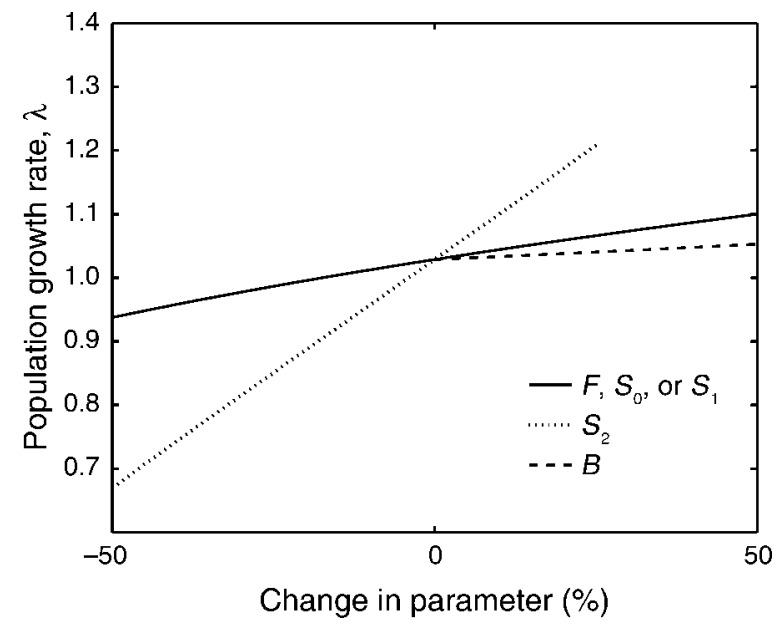

FIG. 1. The largest eigenvalue $\lambda$ vs. change in the falcon life history parameters. Parameters are defined in Table 1.

when that entry is changed and other entries are not changed. These curves are obtained using Eq. A.2 in Appendix A. From these graphs, we see that changes in $S_{2}$ are more important to $\lambda$ than changes in $S_{1}$ or $S_{0}$. Since the long-term growth rate $\lambda$ is most sensitive to $S_{2}$, and $B$ is the most uncertain parameter, we look at how $\lambda$ is affected by simultaneous changes in $B$ and $S_{2}$. In particular, we will determine what changes can be tolerated in $B$ and $S_{2}$ without destroying the conservation property $\lambda>1$.

The traditional approach to analyzing the affect of a change of $p$ to a parameter $a$ on $\lambda$ is via sensitivity analysis. The sensitivity of $\lambda$ to $a$ is the instantaneous rate of change in $\lambda$ with respect to $a$, i.e., it is $d \lambda / d a$ evaluated at the nominal value of $a$ (see Table 1). Even though sensitivity analysis is only guaranteed accurate for small $p$, in this case, the sensitivities in Table 1 lead to the same conclusion as the graphs in Fig. 1.

We now analyze the effect of simultaneous changes in both $B$ and $S_{2}$. We parameterize the change in $B$ by $p_{1}$, and the change in $S_{2}$ by $p_{2}$, where $p_{1}$ is an absolute change and $p_{2}$ is a relative change. In particular, we want the perturbed matrix to be

$$
\tilde{\mathbf{A}}=\mathbf{A}+\mathbf{P}_{1}+\mathbf{P}_{2}=\left[\begin{array}{ccc}
0 & F R S_{1} p_{1} & F R S_{2}\left(1+p_{2}\right) \\
S_{0} & 0 & 0 \\
0 & S_{1} & S_{2}\left(1+p_{2}\right)
\end{array}\right]
$$

As in Appendix A, we write

$$
\mathbf{P}_{1}=p_{1} \mathbf{D}_{1} \mathbf{E}_{1} \quad \mathbf{P}_{2}=p_{2} \mathbf{D}_{2} \mathbf{E}_{2}
$$

where

$$
\mathbf{D}_{1}=\left(\begin{array}{c}
F R S_{1} \\
0 \\
0
\end{array}\right) \quad \mathbf{E}_{1}=\left(\begin{array}{lll}
0 & 1 & 0
\end{array}\right)
$$

and

$$
\mathbf{D}_{2}=\left(\begin{array}{c}
F R S_{2} \\
0 \\
S_{2}
\end{array}\right) \quad \mathbf{E}_{2}=\left(\begin{array}{lll}
0 & 0 & 1
\end{array}\right) .
$$

The admissible range of $p_{1}$ is 0 to 1 , where $p_{1}=1$ implies all two-year-old females breed. The admissible range of $p_{2}$ is constrained so that the term $S_{2}\left(1+p_{2}\right)$, which is a probability, is between 0 and 1 , so $p_{2}$ ranges from -1 to 0.25 . Thus the set of admissible perturbations is described by

$$
S=\left\{\left(p_{1}, p_{2}\right) \mid 0 \leq p_{1} \leq 1,-1 \leq p_{2} \leq 0.25\right\} .
$$

We wish to find the set of $\left(p_{1}, p_{2}\right)$ in $S$ so that $\lambda>1$. We can easily find a curve in the $\left(p_{1}, p_{2}\right)$ plane on which some eigenvalue (not necessarily the largest eigenvalue $\lambda$ ) is equal to one. Hence, on this curve, $\lambda$ must be greater than or equal to 1 . If we can prove that, on this curve, $\lambda=1$, then the curve breaks up the set $S$ of admissible perturbations into two regions, one of which corresponds to $\lambda>1$, while the other region corresponds to $\lambda<1$. In Appendix B, we find the equation of the curve using a method which guarantees that $\lambda=1$ for $\left(p_{1}, p_{2}\right)$ on this curve. The curve is shown in Fig. 2, on a coordinate system with $p_{1}$ on the horizontal axis and $p_{2}$ on the vertical axis. The nominal values of $\left(B, S_{2}\right)$ are represented by $\left(p_{1}, p_{2}\right)=(0,0)$ The shaded area in Fig. 2 represents those $\left(p_{1}, p_{2}\right)$ that correspond to $\lambda>1$.

Fig. 2 shows us how much error is acceptable in $B$ and $S_{2}$, and, more importantly, shows the interplay between uncertainties in the two variables. For instance, for any value of $B, S_{2}$ can tolerate a negative error of $4 \%$ (or, of course, any positive error). If $B=1, S_{2}$ can tolerate a negative error of $13 \%$ or less. This illustrates an important principle: new information about one param-

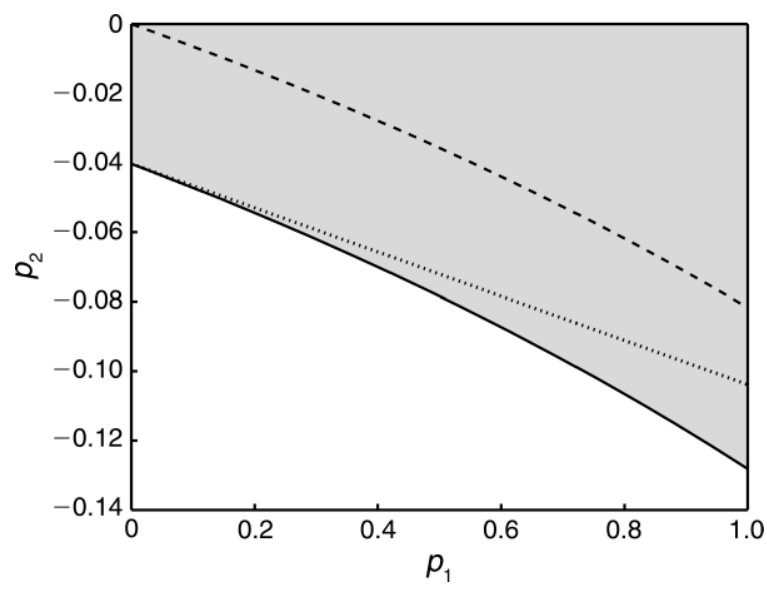

FIG. 2. The boundary curve represents all pairs of perturbations $\left(p_{1}, p_{2}\right)$ for which $\lambda\left(p_{1}, p_{2}\right)=1$. The shaded area represents all pairs of perturbations $\left(p_{1}, p_{2}\right)$ for which $\lambda\left(p_{1}, p_{2}\right)$ $>1$. The dashed line is $\lambda\left(p_{1}, p_{2}\right)=1.0287$, the growth rate of the unperturbed matrix. The dotted line shows the linear approximation to these curves obtained from direct use of sensitivity to predict the effects of perturbations. 


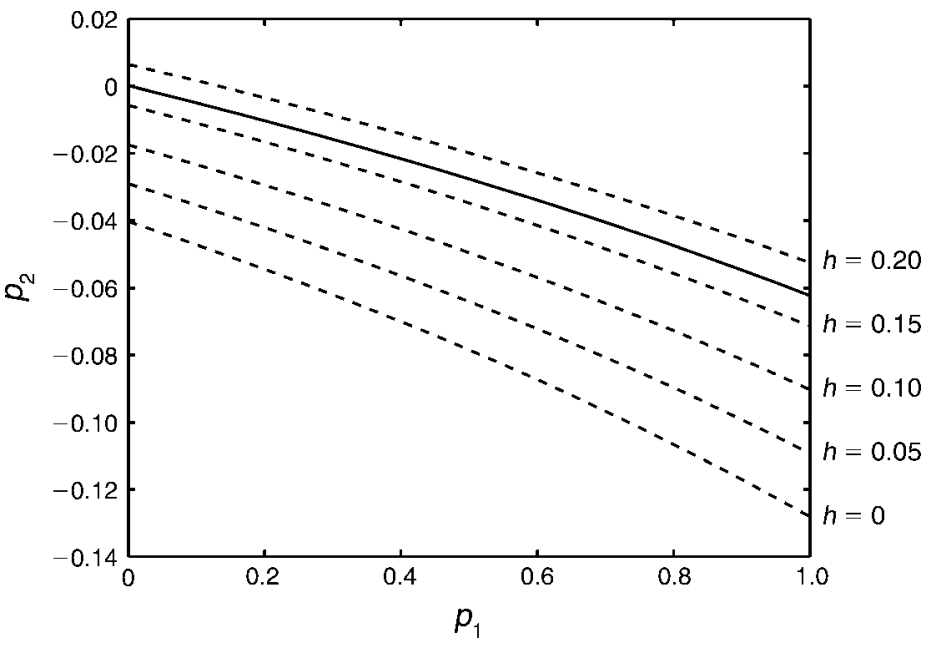

FIG. 3. The effect of the harvesting fraction $h$ on the $\lambda\left(p_{1}, p_{2}\right)=1$ curves. The bold line is $h=$ 0.1714 , the amount of harvesting that yields $\lambda\left(p_{1}, p_{2}\right)=1$ with no uncertainty for the nominal values. eter often changes the robustness to uncertainty in other parameters.

Now suppose that we wish to identify all $\left(p_{1}, p_{2}\right)$ that guarantee a long-term growth rate of at least $3 \%$. Then, we simply replace 1 in our computations with 1.03 . This yields a new curve (Fig. 2) that is shifted upward relative to the previous curve; because $\lambda=1.0287$ at the nominal values, this new curve runs through the nominal point. The region above that curve gives the values of $\left(p_{1}, p_{2}\right)$ for which $\lambda>1.03$ for $\mathbf{A}$.

It is possible to approximate the effect of multiple large perturbations using sensitivities alone by assuming that $\lambda\left(p_{1}, p_{2}\right)$ is linear (Caswell 2001:224; Fig. 2). When uncertainty in $S_{2}$ is considered alone (i.e., along the $y$ axis of the figure), the approximation is very close because the nonlinearity of $\lambda$ with respect to $S_{2}$ is not great (Fig. 1). However, when uncertainty in two parameters is considered simultaneously the linear approximation underestimates how much uncertainty is allowed in $S_{2}$ as $B$ increases. For larger matrices or more complex perturbations, the nonlinearity, and hence the inadequacy of the linear approximation, could easily be more severe.

\section{The effect of harvesting on long-term growth}

We now examine the effect of harvesting on the largest eigenvalue $\lambda$ of the modified population projection matrix $\mathbf{A}_{\boldsymbol{h}}$ (see Eq. 5). As a simple example, let $\mathbf{A}_{\boldsymbol{h}}$ use the nominal values of $B$ and $S_{2}$; we find that the smallest value of $h$ that gives an eigenvalue of 1 is 0.1714 . Therefore, since $\lambda$ varies continuously with $h$ and the nominal matrix A with $h=0$ has largest eigenvalue 1.0288, any value of $h$ less than 0.1714 gives a largest eigenvalue of $\mathbf{A}_{\boldsymbol{h}}$ greater than 1. Thus even with no twoyear-old falcons breeding, if there is no uncertainty, then $17.41 \%$ may be harvested while maintaining a growth rate of $\lambda=1$.

However, this does not take into account uncertainties in $B$ and $S_{2}$. Hence, we again let $p_{1}$ be the uncertainty in $B$ and $p_{2}$ be the uncertainty in $S_{2}$. As in the analysis of $\mathbf{A}$ in Appendix B, for several values of $h$, we find curves in the $\left(p_{1}, p_{2}\right)$ plane on which the largest eigenvalue $\lambda$ for $\mathbf{A}_{\boldsymbol{h}}$ is 1 . For $h=0,0.05,0.1,0.15,0.1714$, and 0.2 , these curves are shown in Fig. 3. The region above each curve gives the values of $\left(p_{1}, p_{2}\right)$ for which $\lambda$ $>1$ for $\mathbf{A}_{\boldsymbol{h}}$. If $B=1$ and $17.41 \%$ are harvested, $S_{2}$ can tolerate uncertainties of up to $-6 \%$. The U.S. Fish and Wildlife Service suggests that $5 \%$ can be harvested. Reading from the $h=0.05$ graph in Fig. 3, we see that, if $B=0$, this allows an uncertainty of $3 \%$ in $S_{1}$, and if $B=$ 1 , this allows an uncertainty of $11 \%$ in $S_{1}$.

If our objective is to maintain $3 \%$ population growth even with harvesting, then we can recalculate our curves as we did for the no-harvesting model (Fig. 2). Although we do not show the figure, it is straightforward to calculate that $3 \%$ population growth cannot be maintained with $5 \%$ harvesting, unless our nominal value of $S_{2}$ is an underestimate, or at least $20 \%$ of two-year-old birds breed. If more than $20 \%$ of two-year-old birds breed, then uncertainties of up to $6 \%$ in adult survival can be tolerated when $B=1$.

\section{DiscusSION}

The difficulty of incorporating the effects of uncertainty in matrix parameters into population management decisions is possibly one of the largest problems preventing widespread adoption of models in decision making. One of the best examples of thoroughly incorporating uncertainty in the assessment of management is Heppell et al.'s (1994) work on Red-cockaded Woodpeckers, which relied on simulation to explore the effects of simultaneous uncertainties, as well as linear approximations using elasticities. This approach of using linear approximations from elasticities in one dimension, and Monte Carlo simulations in multiple dimensions is widely used (e.g., Ferriere et al. 1996, Caswell et al. 1998, among many others). Although it is possible to explore multidimensional parameter uncer- 
tainty reasonably easily in this fashion, the exact results obtained by simulation depend heavily on the details of how perturbations are selected. This is especially true when considering the possibility of constraints or correlations among life history traits; information on such correlations is generally unavailable (Wisdom et al. 2000). Caswell et al. (1998) incorporated constraints on life history traits by sampling survival curves from a group of related species. However, if a different set of species had been selected, the results would differ by an unknown amount, and this still does not answer the problem of correlated environmental variation. The method we introduce here gives an analytical result for all possible perturbations, and is straightforward to implement in readily available software (e.g., Symbolic Toolbox in MATLAB, see Supplement).

It is widely reported that predictions of the perturbations needed to effect a given change in $\lambda$ using sensitivities or elasticities are accurate to relative changes of $\pm 50 \%$ (e.g., de Kroon et al. 2000). However, careful inspection of the numerical examples used to support this claim show that they typically involve perturbations of single vital rates or matrix entries. As shown in Fig. 2 this is true for our matrix as well. However, once multiple parameters are perturbed the linear approximation breaks down. Some examples for multiple perturbations are provided in Caswell (2001; Chapter 18), and these demonstrate increasing approximation errors with both the dimension and size of the perturbation. B. Tenhumberg, S. Louda, J. Eckberg, and M. Takahashi (unpublished manuscript) conducted a Monte Carlo analysis of a large matrix with simultaneous uncertainty in 19 parameters, and found that when parameters varied simultaneously the local and linear elasticities were poor predictors of which parameters have a large influence on $\lambda$. Our method makes all of these predictions easily and without approximation errors.

The notion of using direct perturbations of the life cycle to improve decision making in conservation biology was put forward for empirical perturbations by Ehrlen and van Groenendael (1998). They suggested that the tools of "life table response experiments" (sensu Caswell 2001) should be used to analyze multiple years of data as perturbations of an underlying matrix. A key improvement of this idea over using elasticities alone is the incorporation of the differential variability of each matrix entry (de Kroon et al. 2000), arising because of differential variability in life history traits. However, small observed variation in a vital rate does not necessarily mean it is a poor target for management (Caswell 2001:619), and similarly large observed variation does not automatically lead to a good management target. We have not addressed this issue in the present example, but it would be straightforward to rescale the perturbations $\left(p_{1}, p_{2}\right)$ by the relative amount of variability in the parameters they are affecting, if estimates of this variability are available. A better, prospective approach would rescale the perturbations by their relative cost (ease of manipulation); an excellent example of how to do this using sensitivity analysis is given by Baxter et al. (2006).

A general, but underappreciated, problem with using models to assess the effects of management options is uncertainty in the connection between management and population vital rates. For example, when considering the effects of river flows on fish populations, it may not be at all clear what relationship exists between flow and spawning frequency. This type of uncertainty could be included in the methodology we present here by careful parameterization of the perturbations, although this will increase the number of dimensions in the perturbation, making interpretation more difficult. In the falcon harvesting example we ignored the issue of how many nestlings a harvest rate of 5\% actually represents. There is substantial uncertainty in estimates of numbers of breeding pairs, and consequently in the number of nestlings that can be taken. However, if detectability of breeding pairs is less than 1 , then the actual number of identified nests will be an underestimate. As long as the actual, observed number of nests is used to calculate the number of nestlings that can be taken, the actual harvest rate will be less than $5 \%$. This cannot be guaranteed if the permitted take is based on an estimated number of breeding pairs. In that case, if the breeding population is overestimated then the nominal 5\% harvest rate would in fact be larger, and consequently there is a greater risk that the population growth targets would not be maintained. The robust, conservative decision is to use the actual observed number of nests. This harvest level could be increased, but this is only safe when the accuracy of breeding population estimates can be carefully defined.

We have approached the problem of uncertainty using perturbations in a time-invariant matrix model. Vital rates vary through time and space in natural populations, and ignoring these stochastic effects leads to predictable biases in the long term population growth rates (e.g., Tuljapurkar and Haridas 2006). When comparing management alternatives, the leading eigenvalue of a time invariant matrix works well in the relative sense, because it is a performance measure that integrates across the entire life history (Caswell 2001:615), so for that purpose our approach should work well. Nonetheless it would be an interesting exercise to formally compare the perturbation approach with stochastic population dynamics, and see if they can be combined or reconciled.

Robustness approaches are a relatively new idea in ecology and conservation biology, although they find wide application in many other fields (e.g., Ben-Haim 2001). In addition to applications in conservation biology (e.g., Hodgson and Townley 2004, Hodgson et al. 2006), the concept was recently applied to foraging theory to examine the possibility that foragers seek to guarantee minimum returns rather than maximize 
returns (Carmel and Ben-Haim 2005). The key difference from a decision-making perspective is the shift from maximizing a performance criterion to guaranteeing some minimum level of that criterion. Although our current work focuses on the asymptotic growth rate of a structured population, the general notion of guaranteeing performance could be applied to any measure of how well a population is doing. For example, a minimum probability of quasi-extinction over $T$ years could be specified, and then simulations carried out to determine the largest parameter perturbation that has that as a worst case performance. By restricting our focus to asymptotic population growth rates we enable the use of a powerful set of analytical results rather than having to rely on simulations.

This new approach may make setting objectives for decision making much easier in conservation biology. For example, when comparing two or more management decisions for their effect on the risk of extinction, we may choose the strategy that provides the lowest risk of extinction (Regan et al. 2005). However, if the costs of these decisions differ, we are then forced into making arguments about how much a species is "worth" in order to justify a greater expense. In contrast, if we specify some minimum performance that we wish to guarantee, we can use robustness methods to compare decisions based on how much error each can tolerate and still guarantee the minimum. Differing costs then purchase different levels of robustness, relieving us of the need to value each species. We still have to value the robustness, but this would appear to be easier to do than argue about the value of a species.

In conclusion, the approach we have outlined here provides a powerful set of tools for examining the effect of decisions in the face of large and poorly characterized uncertainty in population projection matrices. Many decisions for threatened and endangered species are made with poor or no information. We can still make decisions under these circumstances in a manner that is highly defensible, even without making assumptions about the distribution of uncertainty or limiting ourselves to discussions of single, infinitesimally small changes in the parameters.

\section{ACKNOWLEDGMENTS}

This work was supported by NSF REU Site Grant 0354008 . R. Rebarber was supported in part by NSF Grant 0206951 . S. Townley was supported in part by a Leverhulme Trust Research Fellowship.

\section{Literature Cited}

Baxter, P. W. J., M. A. McCarthy, H. P. Possingham, P. W. Menkhorst, and N. McLean. 2006. Accounting for management costs in sensitivity analyses of matrix population models. Conservation Biology 20:893-905.

Ben-Haim, Y. 2001. Information-gap decision theory: decisions under severe uncertainty. Academic Press, London, UK.
Carmel, Y., and Y. Ben-Haim. 2005. Info-gap robust-satisficing model of foraging behavior: Do foragers optimize or satisfice? American Naturalist 166:633-641.

Caswell, H. 2001. Matrix population models: construction, analysis, and interpretation. Second edition. Sinauer Associates, Sunderland, Massachusetts, USA.

Caswell, H., S. Brault, A. J. Read, and T. D. Smith. 1998. Harbor porpoise and fisheries: an uncertainty analysis of incidental mortality. Ecological Applications 8:1226-1238.

Craig, G. R., G. C. White, and J. H. Enderson. 2004. Survival, recruitment, and rate of population change of the Peregrine Falcon population in Colorado. Journal of Wildlife Management 68:1032-1038.

de Kroon, H., J. Van Groenendael, and J. Ehrlen. 2000. Elasticities: a review of methods and model limitations. Ecology 81:607-618.

Ehrlen, J., and J. Van Groenendael. 1998. Direct perturbation analysis for better conservation. Conservation Biology 12: 470-474.

Ferriere, R. G., F. Sarrazin, S. Legendre, and J. Baron. 1996. Matrix population models applied to viability analysis and conservation: theory and practice using the ULM software. Acta Oecologica 17:629-656.

Heppell, S. S., J. Walters, and L. B. Crowder. 1994. Evaluating management alternatives for red-cockaded woodpeckers: a modeling approach. Journal of Wildlife Management 58: 479-487.

Hodgson, D. J., and S. Townley. 2004. Linking management changes to population dynamic responses: the transfer function of a projection matrix perturbation. Journal of Applied Ecology 41:1155-1161.

Hodgson, D., S. Townley, and D. McCarthy. 2006. Robustness: predicting the effects of life history perturbations on stagestructured population dynamics. Theoretical Population Biology 70:214-224.

Hunt, W. G. 1988. The natural regulation of peregrine falcon populations. Pages 667-676 in T. J. Cade, J. H. Enderson, C. G. Thelander, and C. M. White, editors. Peregrine Falcon populations: their management and recovery. The Peregrine Fund, Boise, Idaho, USA.

Mills, L. S., D. F. Doak, and M. J. Wisdom. 1999. Reliability of conservation actions based on elasticity analysis of matrix models. Conservation Biology 13:815-829.

Ratcliffe, D. 1993. The Peregine Falcon. Second edition. T and A D Poyser, London, UK.

Regan, H. M., Y. Ben-Haim, B. Langford, W. G. Wilson, P. Lundberg, S. J. Andelman, and M. A. Burgman. 2005. Robust decision-making under severe uncertainty for conservation management. Ecological Applications 15:14711477.

Seneta, E. 1981. Non-negative matrices and Markov chains. Second edition. Springer-Verlag, New York, New York, USA.

Tuljapurkar, S., and C. V. Haridas. 2006. Temporal autocorrelation and stochastic population growth. Ecology Letters 9: 324-334.

U.S. Fish and Wildlife Service. 2001. Falconry take of nestling American Peregrine Falcons in the contiguous United States and Alaska. U.S. Fish and Wildlife Service, Arlington, Virginia, USA.

U.S. Fish and Wildlife Service. 2003. Monitoring plan for the American Peregrine Falcon, a species recovered under the Endangered Species Act. U.S. Fish and Wildlife Service, Divisions of Endangered Species and Migratory Birds and State Programs, Pacific Region, Portland, Oregon, USA.

Wisdom, M. J., L. S. Mills, and D. F. Doak. 2000. Life stage simulation analysis: estimating vital-rate effects on population growth for conservation. Ecology 81:628-641. 
APPENDIX A

Simple perturbations, eigenvalues, and transfer functions (Ecological Archives A017-089-A1).

\section{APPENDIX B}

The $\lambda=1$ curve for two perturbations (Ecological Archives A017-089-A2).

\section{SUPPLEMENT}

MATLAB code to identify the hypersurface (Ecological Archives A017-089-S1). 